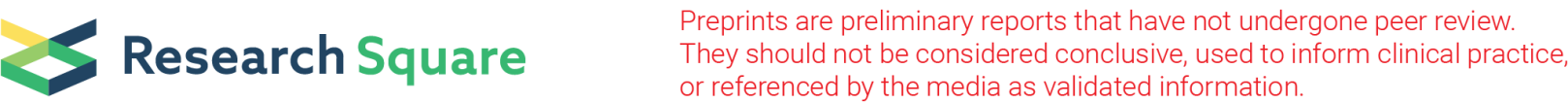

\section{Integrated Multi-Omics Data Analysis Reveals Altered Metabolome Activity and Microbiome Composition in Irritable Bowel Syndrome}

\section{Xiaodong Fang ( $\nabla$ fangxd@bgi.com )}

Guangdong Provincial Hospital of Chinese Medicine https://orcid.org/0000-0001-7061-3337

\section{Lijuan Han}

The Second Affiliated Hospital of Guangzhou University of Chinese Medicine

\section{Ling Zhao}

Hong Kong Baptist University

\section{Yong Zhou}

KMHD

\section{Chao Yang}

KMHD

\section{Teng Xiong}

$\mathrm{KMHD}$

\section{Wen Luo}

KMHD

\section{Yang Chen}

The Second Affiliated Hospital of Guangzhou University of Chinese Medicine

\section{Qinwei Qiu}

The Second Affiliated Hospital of Guangzhou University of Chinese Medicine

\section{Xiaoxiao Shang}

The Second Affiliated Hospital of Guangzhou University of Chinese Medicine

\section{Yusheng Deng}

KMHD

\section{Shaogang Huang}

The Second Affiliated Hospital of Guangzhou University of Chinese Medicine

\section{Suiping Huang}

The Second Affiliated Hospital of Guangzhou University of Chinese Medicine

\section{Zhi Liu}

Huazhong University of Science and Technology

\section{Lin Lu}

Hong Kong Baptist University

\section{Wei Yang}


Hong Kong Baptist University

\section{Lixiang Zhai}

Hong Kong Baptist University

\section{Ziwan Ning}

Hong Kong Baptist University

Chengyuan Lin

Hong Kong Baptist University

\section{Tao Huang}

Hong Kong Baptist University

\section{Chungwah Cheng}

Hong Kong Baptist University

\section{Linda LD Zhong}

Hong Kong Baptist University

\section{Zhaoxiang Bian}

Hong Kong Baptist University

\section{Research}

Keywords: IBS, Metagenome, Metabolome, Multi-omics, Depression.

Posted Date: August 2nd, 2020

DOI: https://doi.org/10.21203/rs.3.rs-51066/v1

License: (c) (1) This work is licensed under a Creative Commons Attribution 4.0 International License. Read Full License 


\section{Abstract}

Background: Irritable bowel syndrome (IBS) is one of functional gastrointestinal disorders mainly characterized by chronic and/or recurrent symptoms of abdominal pain and irregular defecation. Changed gut microbiota has been proposed to mediate IBS; however, contradictory results exist, and the exact mechanism is still debatable. IBS-specific microbiota and metabolite remain poorly understand. To address this issue, we performed untargeted metabolomic and shotgun metagenomic profiling of stool and serum samples from discovery $(n=330)$ and validation $(n=101)$ cohorts of IBS and healthy individuals.

Results: Fecal "omics" data show moderate dysbiosis compared with other disease, in contrast, serum metabolites show significant differences and have great power to discriminate IBS from healthy subjects. Specifically, 726 differentially abundant serum metabolites are identified, including fatty acyl-CoA enriched in IBS. Integrating microbiome and metabolome data, we identified 522 robust associations between differentially abundant species and fecal metabolites, of which three species are strongly associated with the low abundance of dihydropteroic acid. Moreover, the tryptophan enrichment correlates with the severity of IBS depression in both fecal and serum metabolomes.

Conclusions: Collectively, our study unveils serum/fecal metabolome alterations and their relationship with gut microbiome and highlight the massive dysbiosis of serum metabolites which empower to discriminate IBS patients. Our study also provides a valuable resource for future studies to understand host-gut microbiota relationships, and facilitate potential clinical applications using microbiota and (or) metabolites to evaluate IBS patients with depression.

\section{Introduction}

Irritable bowel syndrome (IBS) is a common functional gastrointestinal disorder that affects around $11 \%$ of the population globally[1]. IBS is categorized into four subtypes including diarrhea-predominant IBS (IBS-D), constipation-predominant IBS (IBS-C), mixed IBS (IBS-M) and un-subtyped IBS (IBS-U) according to patients' bowel habits. Moreover, psychological comorbidity including anxiety and depression are common in IBS population[2]. Although the precise mechanism of IBS remains unclear, the alteration of gut microbiota has been proposed to mediate IBS pathogenesis.

Pittayanon and colleagues systematically reviewed gut microbiota studies in IBS from inception to April, 2018, reported the IBS specific intestinal microbiota characterized by an increase in family Enterobacteriaceae, family Lactobacillaceae and genus Bacteroides, together with a decrease in uncultured Clostridiales I, genus Faecalibacterium and genus Bifidobacterium[3]. However, inconsistency results exist among studies, probably due to the differences in methodology, the limited sample size, coupled with the lack of necessary information such as antibiotic/probiotics use and diet habit. For example, one study showed a relative increased abundance of bacterial species including Enterobacteriaceae in feces of IBS patients, with decreased composition in Lactobacillus and 
Bifidobacterium; while opposite results found in other studies described an increase in Lactobacillus genus or Lactobacillales in IBS-D[4]. Tap et.al reported the fecal and mucosal microbiota signatures that related with severity of IBS[5]. Vich et.al reported a large scale of metagenomic sequencing of stool samples from 1792 individuals with IBS and inflammatory bowel disease (IBD), which demonstrate overlapped and specific species between IBD and IBS patients compared with controls[6]. By analyzing the fecal and mucosa-associated microbiome of IBS patients from a Swedish random population, it reported that no IBS specific microbiota feature was identified[7]. However, lan Jeffery et.al reported significant differences in fecal microbiomes and metabolomes of individuals with or without IBS, and highlighted the potential of using metabolomes and microbiome in the diagnosis and treatments of IBS[8].

Microbial composition can shape the environment in the colon as metabolites produced from microbes can be involved in signaling, immune system modulation or have antibiotic activity. For example, shortchain fatty acids (SCFAs) showed lower proportions in serums of IBS patients[9], which play major roles in the microbiota-host interaction by inhibiting inflammatory and malignant growth[10]. A recent study from our group revealed an impaired bile acid synthetic regulation with excessive bile acid excretion contributed by the Clostridia-rich microbiota is associated with the severity of diarrheal symptoms in IBSD patients[11].

These results indicate a potential role of microbiota and microbiota related byproducts in IBS, but the alterations of fecal and serum metabolites and their interactions with gut microbiota are not well established and interpreted. To comprehensively characterize the metagenomics and metabolomics of IBS, in this study, we integrated microbiome and untargeted metabolites profiling data from discovery (n $=330)$ and validation $(n=101)$ cohorts to understand the IBS alterations from healthy patients. We found moderate alteration of fecal microbiome and metabolome between healthy individuals and IBS. In contrast, clear separation between healthy and IBS individuals was observed in serum metabolites. Interestingly, we found tight associations between fecal metabolites and microbiota, for example, the abundance of dihydropteroic acid was associated with many microbial species. Our findings suggest serum metabolites are highly associated with IBS and have valuable insights into IBS pathogenesis.

\section{Results}

\section{Multi-omics profiles associated with demographics and clinical symptoms in IBS population}

To investigate the potential mechanism of pathogenesis in IBS, we collected stool and paired serum samples from a discovery cohort included 264 patients: 24 with IBS-C, 214 with IBS-D, 19 with IBS-M, 7 with IBS-U and 66 healthy controls (Fig. 1a). As shown in Supplementary Table 1, in addition to the abnormal fecal frequency and consistency, nearly 30\% (79 out of 264) of IBS patients also suffered the depression or anxiety symptom. Moreover, some intercorrelations between 24 symptom scores and biochemical indices as well as 45 demographic and dietary factors were identified in this cohort

\section{(Supplementary Figure 1).}


To characterize the microbiome and metabolic profile in IBS, stool samples were subjected to metagenomic sequencing and untargeted metabolomic analysis. Regarding 325 serum samples available, similar high-resolution mass spectrometers were carried out and captured 1769 known and uncharacterized metabolites. We firstly assessed the overall differences between IBS and healthy controls in the fecal microbiome, fecal and serum metabolomes data respectively. Patients with IBS showed elevated alpha and beta diversity in serum and fecal microbiome, without significant differences in fecal metabolome data (Supplementary Figure 2). In addition, we noticed that the major patterns of serum metabolite feature largely separated healthy controls from IBS patients, which is indicative of broad metabolic changes between these two phenotypes (Fig. 1b). Such differences could result from a combination of sources, including the effects of disease activity in host tissues, the activity of IBS-altered microbiome and differences in patient diet and medication use. To explore the origin of serum variations, we further investigated the dysbiosis of gut microbiome, but fewer variations are found among disease phenotypes in the microbial taxonomic profiles and fecal metabolites (Fig. 1b). IBS patients showed a moderate degree of gut microbiota imbalance compared to patients with other microbiota-related diseases (Fig. 1C).

Further, we separately investigated the associations of clinical metrics with metabolic and microbial variations (Fig 1d). Previous studies have suggested that gut microbiota changes are influenced by clinical factors in IBD and IBS[6]. Here, we correlated 24 intrinsic factors and 45 questionnaire factors to the overall composition (Bray-Curtis dissimilarities) and alpha diversity (Shannon index) of microbiome and metabolome. IBS subtypes were identified to be associated with composition changes in microbiome and serum metabolome, but not with fecal metabolome. In microbiome, 10 factors were significantly associated (FDR $<0.1)$ with overall community variation, which together explained $9 \%$ of the variation in interindividual variations (Fig. 1d). Most factors were mutual exclusive in different association analyses, except for fasting glucose. The strongest associations were found for the levels of several biochemical indices with microbial composition, including fecal total bile acid (TBA) and 7a-hydroxy-4-cholesten-3-one (C4), which is consistent with previous studies[12,13]. This trend was also replicated in fecal metabolome in both positive and negative mode (Fig. 1d, Supplementary Figure 3a), which fecal TBA level explained $>6 \%$ of the composition variance that was far greater than any other factors. The fecal TBA levels were negatively associated with fecal metabolomic diversity. In contrast, the Zung Self-Rating Anxiety Scale (SAS) showed the largest association and explained $>8 \%$ of the variance in serum metabolic composition (Fig. 1d, Supplementary Figure 3b), and it was also positively correlated with Shannon diversity. This result demonstrated a close linkage between psychological burden and the change of serum metabolites. Moreover, several dietary factors were also found to be associated with metabolomic results, of which the frequency of tea drinking showed a significant relationship with fecal metabolic variation (Supplementary Figure 3a).

\section{Serum metabolite enrichments in IBS versus control phenotypes.}

To illuminate metabolic changes across groups, we performed partial least-squares discriminant analysis (PLS-DA) on both serum and fecal metabolome data. The serum samples were largely separated between 
IBS and controls, which is consistent with broad changes in serum metabolite profiles described in earlier context (Supplementary Figure $\mathbf{4 b}$ and $5 \mathrm{~b}$ ). By contrast, IBS phenotypes could not be discriminated from controls in fecal metabolome according to PLS-DA plots (Supplementary Figure $6 \mathbf{c}$ and d). We then applied nonparametric univariate method (Wilcoxon rank sum test) to identify differentially abundant metabolites between IBS versus control phenotypes. After correcting for FDR, a total of 726 serum metabolic fragments were significantly changed (FDR < 0.05) in IBS (Supplementary Figure 4a and 5a), whereas only 8 fecal metabolites significantly differed between cases and controls (Supplementary Figure $6 a$ and $b$ ).

Of obtained 726 different metabolic fragments in serum, 101 features were structurally identified (Fig. 2a). Compounds whose levels significantly changed between IBS and controls include many metabolites from food, such as $y$-tocotrienol, myo-Inositol 1-phosphate, stearic acid and actinidine, indicating that some detected changes of metabolite were attributed to the diverse of dietary habits (Fig. $\mathbf{2 b}$ ). Although total serum TBA level increased in IBS (Supplementary Table 1), we observed depletions in bile alcohol 27-Norcholestanehexol and bile salt Taurochenodeoxycholate-3-sulfate. This discrepancy may be due to different enzymes involved in the metabolism and synthesis of different types of bile acids[14]. Another control-enriched metabolite, Tetrahydrodeoxycorticosterone (THDOC), is a stress induced neuroactive and anti-oxidative steroid, which might protect stress-induced responses[15]. This phenomenon is similar to previous study which confirmed the reduced serum concentration of THDOC in women during menstruation epilepsy with depression[16], suggesting it may play a role in the interaction between depression and IBS. Our result showed significant negative correlations between serum level of THDOC with SDS in IBS (Supplementary Figure 7a). On the other hand, IBS-enriched metabolites include Guanine, and four fatty acyl-CoAs, Tetradecanoyl-CoA, Myristoleoyl-CoA, (S)-Hydroxyoctanoyl-CoA and LauroylCoA. Guanine, the most abundant metabolite features in IBS, have been proposed to involve in a specific guanine-based purinergic system which are able to affect development and structure of neural cells in central nervous system and correlated with memory and anxiety[17]. Moreover, fatty acyl-CoA is a group of coenzymes involved in the metabolism of fatty acids. Elevated levels of the three fatty acyl-CoA are consistent with previously reported perturbations of fatty acid metabolism in IBS and major depression[18, 19].

As the simultaneous elevation in IBS of the four fatty acyl-CoAs, we hypothesized that metabolites may be clustered for similar chemical properties. Using the method described by Franzosa et al.[20], 726 differentially abundant metabolites were clustered into 78 clusters which tend to covary independently of their relationship with IBS phenotype and age (Supplementary Table 2). Clusters of metabolites can be used to predict properties for unannotated metabolites by transforming knowledge from their annotated partners. The largest cluster enriched in IBS contained 70 metabolites (Fig. 2c), which include the three of the four fatty acyl-CoAs, enhanced the importance of dysregulation of fatty acids in IBS. Other metabolites in this cluster included some sterol lipids and structural variants of fatty acid. Moreover, 58 unlabeled metabolites were also contained in this cluster which may also be related to fatty acid metabolism via guilt-by-association logic. The largest cluster contained 123 metabolites, and all of them elevated in controls (Supplementary Figure 7b). Validated standard metabolites in this cluster included a 
variety of TG metabolites and phosphates. Another interested cluster enriched in control contained a variety of amines, including Pyridoxamine-5'-Phosphate, Phenylethylamine and Dimethyltryptamine (Fig. 2d). Pyridoxamine 5'-phosphate is one form of vitamin B6, which involved in many reactions of amino acid metabolism[21]. Dimethyltryptamine has a similar chemical structure to the neurotransmitter serotonin and acts as an agonist in mammalian brain and blood[22]. Phenylethylamine is a monoamine neurotransmitter, which can stimulate the body to make certain chemicals that play a role in depression and other psychiatric conditions[23]. The co-functions of these organic compounds in IBS still need further research. Most clusters remained largely undefined, allowing the potential correlation analysis for many previously undescribed metabolites with microbial origin.

\section{Species level changes in IBS microbiome community composition}

Although several previous studies already examined the interaction of gut microbiome and IBS even in large cohort[5, 6], gut microbiome varies dramatically among different geographic population even suffer from same diseases[24], thus worthy investigating in different cohorts. To infer the differences of gut microbiome between IBS and healthy control in Hong Kong populations, we applied LEfSe to the highdimensional taxonomic features. A total of 33 species were differentially abundant, of which 23 were elevated in IBS relative to controls, including Ruminococcus gnavus, Escherichia coli and Bacteroides plebeius. In contrast, Bacteroides uniformis, Prevotella stercorea and Bacteroides coprocola were among the species exhibiting the strongest enrichments in controls (Supplementary Figure 8a, Supplementary Table 3a). IBS patients could display totally different symptom, for example, diarrhea versus constipation, thus important and interesting to figure out the similarities and differences among subtypes in terms of gut microbiome and metabolome. Fig. 3a gives an overview of the gut microbiota differentially identified in all IBS clinical subtypes, depicting the numbers of increased and decreased species per family. In total, 16, 29, 9 and 7 nonredundant taxa were associated with IBS-C, IBS-D, IBS-M and IBS-U patients respectively (Supplementary Figure 8b-e, Supplementary Table 3b-e). Compared with controls, patients with IBS-C or IBS-D showed substantial overlap in the increase and decrease in the relative abundance of bacterial species in their gut microbiome. There were 10 taxa associated with both IBS-D and IBS-C (Supplementary Table 4). These included an increase in several gram-negative bacteria, including Bacteroides faecis, Escherichia coli and Klebsiella pneumoniae. Furthermore, the Bacteroides clarus and Bacteroides coprocola showed an opposite changing direction, may be associated with the different symptoms in IBS-C and IBS-D. It is reported that Bacteroides coprocola showed abnormal distribution of SNPs in T2D patients[25] whereas Bacteroides clarus has been found to be associated with colorectal cancer[26]. In addition, we also found some disease-specific associations. The abundance of Fusobacterium varium, for example, was only elevated in patients with IBS-D but not in those with IBS-C. An increase in species of the Clostridium was observed only in patients with IBS-C, including increases in Clostridium symbiosum and Clostridium bartlettii[11].

To understand the functional consequences of microbial community changes in IBS, we profiled gene families and pathways in all metagenomes using HUMAnN2[27]. The similar LDA Effective Size method was also applied on the abundance data, revealing 3 enzymes and 18 pathways differentially abundant 
in IBS and controls (Supplementary Table 5). Of the differentially abundant pathways, 8 was significantly elevated in IBS patients. It is notable that the TCA cycle III was the most significantly altered pathway, which was dominated by the Bacteroides genus (Fig. 3b), indicating abnormalities in energy metabolism may be involved in IBS. Furthermore, we noticed that the synthesis stearate, androgen and L-tyrosine were enhanced in IBS (Fig. 3c and d, Supplementary Table 5). The elevated tyrosine may increase the sensitivity of tyrosine receptor kinase receptors, which was associated with adjustment of neuronal transmission strength[28]. The most common pathways enriched in healthy controls were associated with biosynthesis of L-lysine (PWY_5097, PWY_2941, PWY_2942 and PWY_724). L-lysine was reported to act like a partial serotonin receptor 4 antagonist and inhibits serotonin-mediated intestinal pathologies and anxiety[29]. Moreover, the biosynthesis of isoleucine and threonine were also enriched in healthy controls (Fig. 3e-g). Our functional analysis revealed differential metabolic pathways between IBS and healthy control, and these observations may help explain the great divergence of serum metabolites in IBS patients.

\section{Associations between metabolites and gut microbiota}

The multi-omics nature of our data enables the identification of dynamic relationship between microbial features and metabolites that are differentially abundant in IBS. Such relationship might be owing to a mechanism that metabolites promote the growth of species or a species produce the metabolites. We analyzed the association between species and fecal/serum metabolites. Interestingly, 522 strong associations $(\mathrm{q}<0.05)$ between differentially gut metabolites and bacteria were revealed $(\mathbf{F i g} .4 \mathrm{a})$, in contrast to which, the association between differentially abundant serum metabolites and bacteria is not strong and did not achieve statistically significance $(q<0.05)$. These findings suggest that serum metabolites are regulated by more complex and strict mechanisms compared to fecal metabolites which could interact directly with gut microbiome. To further enrich for putatively mechanistic relationships that are perturbed in disease, we specifically focused on the subset of associations that were nominally significant ( $p$-value $<0.05$ ) and changes in the same direction between fecal metabolites and bacteria from non-IBS controls. This analysis shows that $30 \%$ (155 out of 522 ) associations could be validated in the controls, including 43 associations involving established metabolites. Of 43 associations, 13 out of 33 differentially abundant species were represented in at least one association. Ruminococcus gnavus associates with 8 metabolites and followed by species Odoribacter splanchnicus and Escherichia coli that associate with 7 metabolites (Supplementary Table 6). In line with IBD, overrepresented abundance of Ruminococcus gnavus is elevated in IBS compared with non-IBS control, and it is negatively associated with dihydropteroic acid, sebacic acid, 2-methyl valeric acid and cortisone. Especially, Ruminococcus gnavus is strongly associated with dihydropteroic acid (Pearson $r=-0.60$ ), which is an important intermediate product for folic acid. Folic acid is reported to be relatively low in IBS patients. Our result demonstrated that the enriched Ruminococcus gnavus was closely related to the low level of folic acid in IBS. In addition, Odoribacter splanchnicus and Escherichia coli are also observed to associate with dihydropteroic acid (Fig. 4b-e, Pearson $r=0.41$ and -0.35 ). Our data demonstrates the importance to integrate gut microbiome and metabolites as it provides an explaining framework to associate microbiome with disease connecting by metabolites and candidates for further investigation. 


\section{Microbial and metabolicsignatures associated with IBS depression}

To investigate potential association between multi-omics signatures and severity of psychic symptoms, we classified samples into four groups: healthy controls (HC), regular IBS without depression (rIBS: HAMD $<7$ or $S A S<50$ or SDS $<53$ ), IBS with mild depression (mIBS: $7 \leq H A M D<17$ and SDS $\geq 53$ ) and IBS with moderate or severe depression (sIBS: HAMD $\geq 17$ and SDS $\geq 53$ ). Partial least-squares discriminant analysis revealed enormous differences between controls and sIBS or mIBS (Fig. 5a). The serum compositional changes involved 836 and 754 analytes that altered in mIBS and sIBS, respectively (Supplementary Table 7). Of them, 693 metabolites are shared. The significantly increased compounds include guanine, stearamide and anandamide. In contrast, we found fewer differences of fecal metabolome between control and depression groups (Fig. 5b, Supplementary Table 8). Similar phenomenon was observed in gut microbiota, and only 37 species showed aberrant alterations (Fig. 5c, Supplementary Table 9). However, functional analysis revealed a gradually increasing enrichment of Ltryptophan biosynthesis pathway across control, rIBS, mIBS and sIBS groups (Fig. 5d, Supplementary Table 10).

To identify metabolic and metagenomic features that distinguish depressed-IBS from regular subjects, we further make a comparison among rIBS, mIBS and sIBS groups. The multi-omics divergence between rIBS and depressed group was smaller than that compared with healthy controls. Notably, the sIBS patients showed more divergence than mIBS patients (Supplementary Figure 9, Supplementary Table 11-13). Interestingly, the L-tryptophan biosynthesis pathway was over-represented in sIBS patient compared with rIBS (Supplementary Table 14). We also quantified depression-related molecules using a targeted metabolic profiling. Consistent with the enhanced TRP biosynthesis ability in gut microbiota, the tryptophan intensity also significantly increased in serum (Fig. 5e). Tryptophan is a precursor to the neurotransmitter serotonin and the increase of tryptophan in serum usually correlates a reduction of neurotransmitter serotonin in brain, which could affect people's mood and cognition. In addition, we also noticed that there are some other elevated compounds in depression group compared with healthy controls, including histamine, tryptamine, kynurenine (KYN) (Supplementary Figure 10).

To explore the associations between neuroactive amino acids/neurotransmitters and gut microbiota, we selected 8 representative species using random forest model (Supplementary Figure 11a and b) and examined their relationships with these molecular compounds. A positive association of Roseburia inulinivorans and histamine was observed in both fecal and serum data (Supplementary Figure 11c), indicating a promoting role of Roseburia inulinivorans in production of histamine. In addition, Roseburia inulinivorans was also associated with melatonin, tryptamine, 3-HAA, kynurenine, glutamine and dopamine in serum, although these associations are not replicated in feces. Furthermore, the IBS-enriched species, Clostridium nexile, correlate with broader range of metabolite changes, such as NAS, TRP, 5-HIAA. Taken together, our data suggested that gut microbiome producing amino acids and amines, such as tryptophan, serotonin and histamine, could be involved in synthesis and degeneration of many neurotransmitters thus affecting host's mode and psychological conditions. The integration of 
metagenome and metabolome data enable us to connect the bacteria and its associated metabolic products to partly explain the depression in IBS patients.

\section{Multi-omics features differentiate IBS subtypes from controls}

Diagnosis and classification of IBS subtypes are currently achieved mainly by a symptom-based manner. To investigate the possibility of developing classifiers based on gut microbiome and/or metabolites, we build a random forest model using the filtered relative abundance of species feature and 10-fold cross validation in our discovery cohort with an independent cohort as validation.

AUC of IBS versus control is 0.839 (95\% Cl: $79.2 \%-88.69 \%)$ and $0.639(95 \% \mathrm{Cl}: 58.93 \%-68.99 \%)$ in the independent validation cohort containing 73 samples (Fig. 6a). Using the similar procedure above, we find that the AUC of IBS-D versus control is 0.855 (95\% Cl: $80.81 \%-90.14 \%)$ in discovery cohort, and 0.746 (95\% Cl: $70.52 \%-78.6 \%)$ in the independent validation cohort (Supplementary Figure 12a and b). The same procedure is applied to IBS-C, IBS-M and IBS-U. But only IBS-C result is shown since the number of other subtypes is limited. In line with Shankar's result[30], classification performance using fecal metabolome data presents an AUC of 0.882 (95\% Cl: $83.37 \%-92.93 \%)$ in 10 fold cross validation and AUC of 0.709 (95\% Cl: $64.33 \%-77.46 \%$ ) in an independent validation set (IBS=32 and control=7) (Fig. 6b). Similar trend could be observed in IBS-D versus control model, achieving AUC of 0.880 (95\% Cl: $82.66 \%$ $-93.29 \%$ ) in discovery cohort and of 0.671 (95\% Cl: $60.08 \%-74.15 \%)$ in validation cohort (IBSD=18, control=7) (Supplementary Figure $12 \mathrm{c}$ and $\mathrm{d}$ ).

As serum metabolome data showed great divergence between IBS patients and controls, similar random forest procedure was applied as well. IBS disease is almost perfectly classified from control with AUC of 0.997 (95\% Cl: 99.17\%-100\%) (Fig. 6c) in discovery cohort and of 0.998 (95\% Cl: 99.67\%-99.95\%) in validation cohort. IBS-D disease could be superiorly predicted from control as well, achieving AUC of 0.996 (95\% Cl: $99.15 \%-100 \%)$, and unfailingly of 0.997 (95\% Cl: $99.49 \%-99.92 \%)$ in validation cohort (IBS-D=57, control=15) (Supplementary Figure 12e and f). Similar trends could be replicated in IBS-C, IBS$M$ and IBS-U despite limited sample numbers. All these supervised learning results are consistent with our unsupervised PCoA analysis of serum metabolites (Supplementary Figure 4c) that IBS disease could be well distinguished from control. Since the timeline of clinical medication and blood sampling is not recorded, the metabolomic discrepancy could be consequence of drug metabolism, and larger cohort data with detailed record are needed to validate our speculation. However, we only find mediocre performance of metabolome data among inter-subtypes models, such as the IBS-D versus IBS-C (Supplementary Figure 12e and f).

Since IBS has been found to be accompanied by anxiety and depression, attempts using microbiota to differentiate different depression status has been put into practice[31]. In our data, AUC of predicting presence and absence of depression in IBS using microbiota data is $69.1 \%$ (95\% Cl: $62.31 \%-75.89 \%)$ (Fig. 6d). Stratifying into rIBS (regular IBS, $n=162$ ), mIBS (IBS with mild depression; $n=81$ ) and sIBS (IBS with serious depression; $\mathrm{n}=17$ ) subgroups, AUC of each subgroup predicting from control is more than 0.8 (Supplementary Figure 13a). Specifically, model built based on the rIBS and mIBS subgroups and tenfold 
cross validation has an AUC value of 0.639 (95\% Cl: $56.76 \%-70.96 \%)$, indicating that microbiota shift between rlBS and mIBS might be weak (Supplementary Figure 13b). Using similar procedure, we sought to build a model using the metabolomic data and similar trends could be observed in fecal untargeted data (Fig. 6e, Supplementary Figure 13c and d). In the serum metabolomic data, only $72.4 \%$ (95\% Cl: $65.73 \%-79.07 \%$ ) and $69.09 \%$ (95\% Cl: $62.24 \%-75.93 \%)$ of AUC could be reached in positive and negative mode for predicting presence and absence of depression in IBS, respectively (Fig. 6f). All the rIBS $(n=162)$, mIBS $(n=81)$ and sIBS $(n=17)$ could be well differentiated from control, achieving AUC of 0.990 and higher (Supplementary Figure 13e). These results indicate that metabolome data might not be well in predicting depression status although it could clearly separate IBS patients from health control.

\section{Validation of differentially abundant microbiome and metabolites}

We evaluated the generality of the differentially abundant serum metabolites identified earlier in the discovery cohort. Of 726 serum metabolites that were differentially abundant in IBS, 635 (87.5\%) showed in the same enrichment in the validation cohort, of which 628 were also FDR significant (Supplementary Table 15). In microbiome data, 24 of 33 species that were differentially abundant in discovery cohort trended in the same direction with only 2 achieving statistical significance. Taken together, the majority of IBS-associated changes identified in the discovery cohort generalized in their directionality to validation cohort especially for metabolites.

\section{Discussion}

This study reports large-scale integrated analysis of microbiome and fecal/serum metabolome of IBS individuals for the first time. Compared with other diseases' relationship with microbiome, IBS shows smaller changes in both gut microbiome and fecal metabolome from healthy controls, and these findings could be confirmed by other studies that no clear separation of IBS and healthy control was observed based on gut microbiota. Although we did not observe significant changes in microbiome and metabolic diversity between IBS and healthy controls, we did notice some differentially abundant species such as Ruminococcus gnavus, Escherichia coli and Bacteroides plebeius. These results prompt us to anticipate that IBS is a disease with moderate alterations in fecal microbiota and metabolites. Moreover, tea drinking was observed to be associated with fecal microbiota and metabolites in IBS, which is consistent to previous study that tea could have effect on gut microbiota[32]. However, more detail relationship between tea and IBS are needed in further research.

In contrast to fecal microbiome and metabolome, serum metabolites demonstrated clearly separation of IBS from healthy controls. Our data revealed that nearly $50 \%$ serum metabolites exhibit significantly changes, and these data might provide important implications for IBS diagnosis. Less than $15 \%$ of differentially abundant serum metabolites were structurally qualified, indicating the limitation of untargeted metabolomics approaches. Therefore, we applied a clustering method based on covariation of residual across samples to identify metabolite clusters with similar physico-chemical properties. Such clusters may include metabolites with correlated pro-inflammatory effects and, are needed for future 
experimental characterization. Specially, the largest IBS-enriched cluster included three fatty acyl-CoA which involved in the metabolism of fatty acids. This clustering of metabolites may play a role in dysregulation of fatty acid profile in IBS. Chua et al. ${ }^{10}$ showed omega-3 polyunsaturated fatty acids deficiency in IBS which was ascribed to substantial effects on the nervous systems. Clarke et al.[33] suggested omega-3 supplementation may be a putative treatment for IBS. Our result may help to establish the broad efficacy of omega-3 in improving IBS. However, the precise diagnosis of IBS from other intestinal-related diseases need further comparison of serum profiling from different types of intestinal disease such as IBD to identify the IBS-specific serum metabolites.

Strong associations are observed between fecal microbiota and metabolites compared to serum metabolites. These findings are consistent with the notion that gut microbiome produces and interacts with fecal metabolites directly. Interestingly, we found species associated with dihydropteroic acid, which is an intermediate product for folic acid. The deficiency of folic acid is previously reported in IBS[34], and the supplementation of folic acid is effective in relieving IBS. As 11 out of 33 differentially abundant species are associated with dihydropteroic acid, suggesting IBS-featuring microbiome could affect disease by modulating metabolism of folic acid. Further studies need to confirm the casual relationship and biological mechanism between the function of these species and folic acid.

Analyses of IBS patients with depression also provide profiling of trans-omics features of depressed IBS patients. A great divergence in serum metabolites were observed in the depressed IBS compared with healthy controls, whereas this separation largely reduced in fecal data. Functional analysis of fecal microbiota revealed several pathways enriched in depression group, including the biosynthesis of dTDP$\mathrm{N}$-acetylviosamine, L-glutamate, L-glutamine, tetrahydrofolate, tetrapyrrole and L-tryptophan. Notably, the abundance of L-tryptophan pathway was gradually elevated from control, rIBS to mIBS and sIBS group, suggesting it may play important role in IBS with depression. Further identification of depression associated molecular neurotransmitters using target LC-MS also verified the increased signal intensity of tryptophan in serum. It is well known that the tryptophan is the precursor of serotonin, and the increase of tryptophan will induce the upregulation of serotonin in blood and downregulation in brain, which could affect people's mood. The irregularity of tryptophan in serum was noticed in IBS-D[35], but its role in IBSinduced depression and associated gut microbiome alterations remain elusive. Our correlation analysis of molecular neurotransmitters and gut microbiota revealed underlying associations between tryptophan and Clostridium nexile. Clostridium nexile could produce neurotransmitter tryptamine, and some other Clostridium species, such as $C$. Diffcile, has been reported to increase in patients with major depressive disorder[36, 37]. Further animal experiment will need to investigate whether the increase of Clostridium nexile induce the dysregulation of tryptophan in blood.

Finally, IBS is a collection of gastrointestinal syndromes with potential functional disorder, no clear pathogenesis was observed in a certain organ. It might be caused by many distinguished factors thus consistent alternations of gut microbiome and fecal metabolites could not be eagerly expected, as exampled by our data and previous studies. Unexpectedly, we observed significantly differences of serum metabolites in large scale between IBS patients and health controls, leading to a hypothesis that IBS 
might be highly associated with metabolic disorder. Although most of the metabolites could not be clearly identified due to the limitation and untargeted nature of mass-spectrum technology, some known depression associated neurotransmitters were found to be differential between patients and controls and associated with depression syndrome. Additionally, our functional analysis indicated gut microbiome metabolic pathways abundance shifted between IBS and normal control, for example the energy associated TCA cycle pathway, and these observations provide an evidence of metabolic disorder in IBS. Indeed, previously population-based cross-sectional study in IBS had found it was positively relative to metabolic syndrome[38], reinforcing the hypothesis that IBS could be ascribed to metabolic disorder, but more attentions and evidences are required for this topic.

\section{Methods}

\section{Sample collection}

Adults meeting the Rome IV criteria for IBS[39, 40] were prospectively recruited at two Chinese medicine clinics affiliated with the School of Chinese Medicine, Hong Kong Baptist University. In discovery cohort, we collected 330 fecal tissues and 326 of them had matched serum samples. The disease status was classified by predominant bowel habits on the days with abnormal bowel movements based on the questionnaire of Bristol Stool Form scale and defecation frequency[39]. Matched healthy volunteers without medical history or neurodegenerative diseases, cardiovascular diseases, metabolic disorders, GI diseases and surgical histories of GI tract, cerebral cranium and gallbladder removal were also recruited in the same clinical centers. Included subjects were instructed to provide morning first stool samples and fasting blood samples for biochemical detection and omics analyses. All included participants were required to stop using antibiotics, probiotics, prebiotics and other microbiota-related supplements at least three weeks before stool sampling. Specimens (serum and stools) were transported to the laboratory using dry ice and were frozen at $-80^{\circ} \mathrm{C}$.

\section{Approval for human patient research}

This study was approved by the Ethics Committee on the Use of Human \& Animal Subjects in Teaching \& Research (Approval no. HASC/15-16/0300 and HASC/16-17/0027). Written informed consent was obtained from each participant prior to sample collection. Patients were not involved in this research. Patients were not invited to comment on the study design or interpret the results. Patients were not invited to contribute to the writing or editing of this manuscript.

\section{Measurements of total BA, C4 and FGF19 in serum samples}

Concentrations of total BA and FGF19 in human serum were tested by using commercial Total Bile Acid Assay Kit (Cell Biolabs, San Diego, CA, USA) and Human Fibroblast growth Factor 19 Assay Kit (Thermo scientific, Waltham, MA, USA), respectively. The level of serum C4 was quantified by a liquid chromatography coupled with mass spectrometry (LC/MS)-based method developed from our group. 


\section{Metabolite profiling of fecal and serum samples}

Metabolites were extracted from serum and fecal samples as described previously[18, 41]. Briefly, feces (100 mg) were completely homogenized with five-fold volume of ice-cold distilled water. After high-speed centrifugation (13,000 rpm for $15 \mathrm{~min}$ at $\left.4{ }^{\circ} \mathrm{C}\right)$, water extractions were transferred to a new 2-mL tube. Subsequently, another five-fold volume $(500 \mu \mathrm{L})$ of methanol was added into the pellet sample. The mixture was completely homogenized and centrifuged again. Methanol extractions were combined with the previous water extractions. Serum $(50 \mu \mathrm{L})$ was prepared with four volumes of cold methanol for protein precipitation, and metabolite extracts were obtained after vortex and centrifugation. The $200 \mu \mathrm{L}$ of fecal or serum supernatant was dried and redissolved in the same volume of solvent consisting of water and acetonitrile (98:2, v/v). Meanwhile, quality control (QC) samples pooling all samples were individually prepared using the same protocol. P-chlorophenylalanine $(5 \mu \mathrm{g} / \mathrm{mL})$ was added as an internal standard.

\section{Analytical conditions for the untargeted metabolic profiling}

The $2 \mu \mathrm{L}$ of resulting supernatant was injected into a liquid chromatography system (UPLC, Agilent 1290 Infinity, USA) and separated by gradient elution with $0.35 \mathrm{~mL} / \mathrm{min}$ of flow rate using ACQUITY UPLC BEH C18 column $(1.7 \mu \mathrm{m}, 2.1 \times 50 \mathrm{~mm}$, Waters Corporation, Milford, MA). The gradient program consisted of phase $A(0.1 \%$ formic acid in water) and phase $B(0.1 \%$ formic acid in acetonitrile), which started from 2 to $5 \% \mathrm{~B}$ in $1 \mathrm{~min}$, then raised to $100 \% \mathrm{~B}$ in next $11 \mathrm{~min}$ and maintained at $100 \% \mathrm{~B}$ for 3 min, finally turned back to $2 \%$ B in 2 min. A quadruple time-of-flight mass spectrometer (Q-TOF/MS, Agilent 6543, USA) coupled with electrospray ionization (ESI) was performed for acquisition of metabolic fragments in both positive and negative ionic modes. The instrument operated in full scan mode from 100 to $1,000 \mathrm{~m} / \mathrm{z}$ and the capillary voltage was set at $3,000 \mathrm{~V}$.

\section{Semi-quantification of neuroactive amides in feces and serum}

A total of 17 neuroactive metabolites including tryptophan (TRP), tryptamine (TRPT), n-acetylserotonin (NAS), 5-hydroxyindoleacetic acid (5-HIAA), melatonin, kynurenine (KYN), kynurenic acid (KYA), serotonin, 3-indole acetic acid (3-IAA), 3-hydroxyanthranilic acid (3-HAA), tyrosine (TYR), succinic acid (SUCC), dopamine, glutamate (GLU), glutamine (GLN), histamine, aminobutyric acid (GABA) were purchased from Sigma-Aldrich (St. Louis, MO, USA). An isotopic glutamine-2,3,3,4,4-d5 as internal standard was obtained from CDN isotopes (Pointe-Claire, Quebec, Canada). HPLC grade organic reagents for mass spectrometric analysis were purchased from Sigma-Aldrich (St. Louis, MO, USA). The standard curves and regression coefficients were gained based on IS adjustment.

The analytical conditions were referred to a published study[42]. Briefly, a liquid chromatography (Agilent UHPLC 1290, USA) coupled with a triple-quadrupole mass spectrometer (Agilent QQQ-MS 6438, USA) was applied. Sample injection and flow rate were set at $2 \mu \mathrm{L}$ and $0.4 \mathrm{ml} / \mathrm{min}$ for each sample, respectively. Of neuroactive metabolites, GLU, GLN, histamine, GABA were separated using a ACQUITY BEH Amide column $(1.7 \mu \mathrm{m}, 100 \mathrm{~mm} \times 2.1 \mathrm{~mm})$ with a linear gradient of $100 \mathrm{mM}$ ammonium formate in $95 \%$ water and $5 \%$ acetonitrile (mobile phase $\mathrm{A}$ ) and $30 \mathrm{mM}$ ammonium formate in $15 \%$ water and $85 \%$ acetonitrile 
(mobile phase B). The gradient program was: $100 \%$ to $80 \%$ B for the first $6 \mathrm{~min}, 80 \%$ to $50 \% \mathrm{~B}$ for $3 \mathrm{~min}$, held at $50 \%$ B for $3 \mathrm{~min}, 50 \%$ to $100 \% \mathrm{~B}$ for $3 \mathrm{~min}$. The column temperature was maintained at $30^{\circ} \mathrm{C}$. Moreover, melatonin, NAS, KYN, KYA, TRP, 5-HIAA, TYR, serotonin, 3-IAA, TRPT, 3-HAA, dopamine, SUCC were separated using a ACQUITY BEH C18 column $(1.7 \mu \mathrm{m}, 100 \mathrm{~mm} \times 2.1 \mathrm{~mm})$ with a linear gradient of $0.1 \%$ formic acid $(F A)$ in water (mobile phase $A$ ) and $0.1 \%$ FA in acetonitrile (mobile phase $B$ ). The gradient program was: $2 \%$ to $30 \%$ B for the first $4 \mathrm{~min}, 30 \%$ to $100 \%$ B for $2 \mathrm{~min}$, held at $100 \%$ B for 2 min, $100 \%$ to $2 \%$ B for $2 \mathrm{~min}$. The column temperature was maintained at $40^{\circ} \mathrm{C}$. The capillary voltage of mass spectrometer for both acquisitions was $3.5 \mathrm{kV}$ in the positive mode. The acquisition data was analyzed using Agilent MassHunter Workstation Software for peak integration, calibration equations and quantification of individual metabolites.

\section{Identification of differentially abundant metabolites}

The nonparametric univariate method (Wilcoxon rank sum test) was applied to identify metabolites that differed in abundance between IBS patients and controls and corrected for false discovery rate (FDR). Partial least-squares discriminant analysis (PLS-DA) were applied by using the function plsda from the $R$ package mixOmics. Metabolites which were significantly differential abundant between IBS and controls were identified on the basis of variable importance in the projection (VIP) threshold of 1 from the tenfold cross-validated PLS-DA model, which also passed Wilcoxon rank sum test with adjusted $P<0.05$, fold change $<0.8$ or $>1.2$.

\section{Unsupervised clustering of differentially abundant metabolites}

To calculate residual abundance values, we first log-transformed the relative abundance values to variance-stabilize the data. Zero values were additively smoothed by half the smallest non-zero measurement on a per-sample basis. We then modelled the transformed abundance of each feature as a function of IBD phenotype (modelled as a categorical variable), with age as a continuous covariate. Residual abundance values from the linear models were used in subsequent cluster analyses.

We performed clustering on residual abundance values of all differentially abundant metabolites. This procedure enriches for covariation between metabolites. Metabolites were first ranked according to the richness in IBD (the fold change). The highest-ranked metabolite was assigned as an initial cluster. Each subsequent metabolite was then compared to each extant cluster. If the metabolite had a mean similarity to the cluster's members exceeding a threshold, the metabolite was added to that cluster (We applied Spearman's rank correlation with a threshold of $r=0.7$ ). If the metabolite was not added to any extant clusters, it was seeded into a new cluster. After scanning all metabolites, clusters were renumbered according to their size, of which cluster 1 had the largest size, and so on.

\section{Metagenomic DNA extraction and sequencing}

We used the phenol/chloroform/isoamyl alcohol method to extract microbial DNA from stool samples (200 mg) of included subjects[43]. The DNAs that passed quality control were then broken into library 
using the TruSeq DNA HT Sample Prep Kit. Paired Sequencing were carried out on Illumina platform (read length 150).

\section{Read-level quality control and metagenomic profiling}

Raw sequencing reads were filtered with SOAPnuke[44]. Host (human) reads were identified and removed by mapping against the human genome (hg19 build) with SOAP2[45]. The remaining high-quality reads were used for further analysis. MetaPhIAn2[46] was used to estimate the relative abundance of taxonomic profile. We only considered the species-level data and kept species that exceed $0.1 \%$ relative abundance in at least six samples. Functional profiling was performed using HUMAnN2[27] version 0.9.4 in UniRef90 mode. The gene family and pathway level outputs are produced in reads per kilobase units and then sum-normalized to ppm units. LEFSE[47] analysis was applied on the relative abundance of species, gene families and pathways to identify disease associated biomarkers.

\section{Statistical analysis}

We assessed how many variations of Bray-Curtis distance of microbiome and metabolome can be explained by clinical factors using the function adonis from the $\mathrm{R}$ (3.5.1) vegan package. The $\mathrm{P}$ value was determined by 1000x permutations and was further adjusted using the Benjamini and Hochberg method. The association between each factor and each diversity or richness measure was assessed by Spearman correlation. The 10-fold cross-validation random forest model was generated using $\mathrm{R}$ (3.5.1) randomForest package. The cross-validational error curves from 5 trials of the 10-fold cross-validation were averaged, and the minimum error in the averaged curve plus the standard error at that point was used as the cutoff. The minimum number of species/metabolites markers with an error less than the cutoff was chosen as the optimal model. The ROC curves were drawn using R (3.5.1) pROC package.

\section{Declarations}

\section{Availability of data and material}

Fecal metagenomic sequencing reads can be downloaded from CNGB Nucleotide Sequence Archive (https://db.cngb.org/cnsa/) under accession number CNP0000334.

\section{Acknowledgements}

Not Applicable.

\section{Funding}

This study was supported by the National Natural Science Foundation of China (81672818), the Project of State Key Laboratory of Dampness Syndrome of Chinese Medicine, Science Technology and Innovation Committee of Shenzhen Municipality under grant No. JCYJ20160331190123578, and the Innovation and Technology Fund, Hong Kong SAR (ITS-148-14FP). 
LJH, YZ, CY, TX, WL, YC, QWQ, XXS and YSD analyzed the data; LJH, LZ, CY, TX, SGH, SPH and ZL interpreted the data; $L Z, L L, W Y, C Y L, T H, L X Z$ and $Z W N$ collected samples and conducted the experiments; CWC, LLZ and ZXB recruited patients; LJH, CY, TX, ZCM and YZ wrote the paper. LZ, ZXB and XDF revised the paper. XDF designed and supervised the project. All authors reviewed and approved its final version.

\section{Ethics declarations}

\section{Ethics approval and consent to participate}

This study was approved by the Ethics Committee on the Use of Human \& Animal Subjects in Teaching \& Research (Approval no. HASC/15-16/0300 and HASC/16-17/0027). Written informed consent was obtained from each participant prior to sample collection.

\section{Consent for publication}

Not applicable.

\section{Competing interests}

The authors declare that they have no competing interests.

\section{References}

1. Lovell RM, Ford AC: Global prevalence of and risk factors for irritable bowel syndrome: a metaanalysis. Clinical gastroenterology and hepatology : the official clinical practice journal of the American Gastroenterological Association 2012, 10(7):712-721 e714.

2. Zamani M, Alizadeh-Tabari S, Zamani V: Systematic review with meta-analysis: the prevalence of anxiety and depression in patients with irritable bowel syndrome. Alimentary pharmacology \& therapeutics 2019, 50(2):132-143.

3. Pittayanon R, Lau JT, Yuan Y, Leontiadis GI, Tse F, Surette M, Moayyedi P: Gut Microbiota in Patients With Irritable Bowel Syndrome-A Systematic Review. Gastroenterology 2019, 157(1):97-108.

4. Rodino-Janeiro BK, Vicario M, Alonso-Cotoner C, Pascua-Garcia R, Santos J: A Review of Microbiota and Irritable Bowel Syndrome: Future in Therapies. Advances in therapy 2018, 35(3):289-310.

5. Tap J, Derrien M, Tornblom H, Brazeilles R, Cools-Portier S, Dore J, Storsrud S, Le Neve B, Ohman L, Simren M: Identification of an Intestinal Microbiota Signature Associated With Severity of Irritable Bowel Syndrome. Gastroenterology 2017, 152(1):111-123 e118.

6. Vich Vila A, Imhann F, Collij V, Jankipersadsing SA, Gurry T, Mujagic Z, Kurilshikov A, Bonder MJ, Jiang $\mathrm{X}$, Tigchelaar EF et al: Gut microbiota composition and functional changes in inflammatory bowel disease and irritable bowel syndrome. Science translational medicine 2018, 10(472). 
7. Hugerth LW, Andreasson A, Talley $\mathrm{NJ}$ : No distinct microbiome signature of irritable bowel syndrome found in a Swedish random population. Gut 2020, 69(6):1076-1084.

8. Jeffery IB, Das A, O'Herlihy E, Coughlan S, Cisek K, Moore M, Bradley F, Carty T, Pradhan M, Dwibedi C et al: Differences in Fecal Microbiomes and Metabolomes of People With vs Without Irritable Bowel Syndrome and Bile Acid Malabsorption. Gastroenterology 2020, 158(4):1016-1028.e1018.

9. Undseth R, Jakobsdottir G, Nyman M, Berstad A, Valeur J: Low serum levels of short-chain fatty acids after lactulose ingestion may indicate impaired colonic fermentation in patients with irritable bowel syndrome. Clinical and experimental gastroenterology 2015, 8:303-308.

10. Guilloteau P, Martin L, Eeckhaut V, Ducatelle R, Zabielski R, Van Immerseel F: From the gut to the peripheral tissues: the multiple effects of butyrate. Nutrition research reviews 2010, 23(2):366-384.

11. Zhao L, Yang W, Chen Y, Huang F, Lu L, Lin C, Huang T, Ning Z, Zhai L, Zhong LL et al: A Clostridiarich microbiota enhances bile acid excretion in diarrhea-predominant irritable bowel syndrome. The Journal of clinical investigation 2020, 130(1):438-450.

12. Wong BS, Camilleri M, Carlson P, McKinzie S, Busciglio I, Bondar O, Dyer RB, Lamsam J, Zinsmeister AR: Increased bile acid biosynthesis is associated with irritable bowel syndrome with diarrhea. Clinical gastroenterology and hepatology : the official clinical practice journal of the American Gastroenterological Association 2012, 10(9):1009-1015.e1003.

13. Vijayvargiya P, Busciglio I, Burton D, Donato L, Lueke A, Camilleri M: Bile Acid Deficiency in a Subgroup of Patients With Irritable Bowel Syndrome With Constipation Based on Biomarkers in Serum and Fecal Samples. Clinical gastroenterology and hepatology : the official clinical practice journal of the American Gastroenterological Association 2018, 16(4):522-527.

14. Wahlström A, Sayin SI, Marschall HU, Bäckhed F: Intestinal Crosstalk between Bile Acids and Microbiota and Its Impact on Host Metabolism. Cell metabolism 2016, 24(1):41-50.

15. Wetzel $\mathrm{CH}$, Vedder $\mathrm{H}$, Holsboer F, Zieglgansberger W, Deisz RA: Bidirectional effects of the neuroactive steroid tetrahydrodeoxycorticosterone on GABA-activated $\mathrm{Cl}$ - currents in cultured rat hypothalamic neurons. British journal of pharmacology 1999, 127(4):863-868.

16. Tuveri A, Paoletti AM, Orru M, Melis GB, Marotto MF, Zedda P, Marrosu F, Sogliano C, Marra C, Biggio $\mathrm{G}$ et al: Reduced serum level of THDOC, an anticonvulsant steroid, in women with perimenstrual catamenial epilepsy. Epilepsia 2008, 49(7):1221-1229.

17. Schmidt AP, Lara DR, Souza DO: Proposal of a guanine-based purinergic system in the mammalian central nervous system. Pharmacology \& therapeutics 2007, 116(3):401-416.

18. Chua CS, Huang SY, Cheng CW, Bai CH, Hsu CY, Chiu HW, Hsu JL: Fatty acid components in Asian female patients with irritable bowel syndrome. Medicine 2017, 96(49):e9094.

19. Mamalakis G, Tornaritis M, Kafatos A: Depression and adipose essential polyunsaturated fatty acids. Prostaglandins, leukotrienes, and essential fatty acids 2002, 67(5):311-318.

20. Franzosa EA, Sirota-Madi A, Avila-Pacheco J, Fornelos N, Haiser HJ, Reinker S, Vatanen T, Hall AB, Mallick H, Mclver LJ et al: Gut microbiome structure and metabolic activity in inflammatory bowel disease. Nature microbiology 2019, 4(2):293-305. 
21. Merrill AH, Jr., Henderson JM: Vitamin B6 metabolism by human liver. Annals of the New York Academy of Sciences 1990, 585:110-117.

22. Smith RL, Canton H, Barrett RJ, Sanders-Bush E: Agonist properties of N,N-dimethyltryptamine at serotonin 5-HT2A and 5-HT2C receptors. Pharmacology, biochemistry, and behavior 1998, 61(3):323330.

23. Szabo A, Billett E, Turner J: Phenylethylamine, a possible link to the antidepressant effects of exercise? British journal of sports medicine 2001, 35(5):342-343.

24. He Y, Wu W, Zheng HM, Li P, McDonald D, Sheng HF, Chen MX, Chen ZH, Ji GY, Zheng ZD et al: Regional variation limits applications of healthy gut microbiome reference ranges and disease models. Nature medicine 2018, 24(10):1532-1535.

25. Chen Y, Li Z, Hu S, Zhang J, Wu J, Shao N, Bo X, Ni M, Ying X: Gut metagenomes of type 2 diabetic patients have characteristic single-nucleotide polymorphism distribution in Bacteroides coprocola. Microbiome 2017, 5(1):15.

26. Liang Q, Chiu J, Chen Y, Huang Y, Higashimori A, Fang J, Brim H, Ashktorab H, Ng SC, Ng SSM et al: Fecal Bacteria Act as Novel Biomarkers for Noninvasive Diagnosis of Colorectal Cancer. Clinical cancer research : an official journal of the American Association for Cancer Research 2017, 23(8):2061-2070.

27. Franzosa EA, Mclver LJ, Rahnavard G, Thompson LR, Schirmer M, Weingart G, Lipson KS, Knight R, Caporaso JG, Segata $\mathrm{N}$ et al: Species-level functional profiling of metagenomes and metatranscriptomes. Nature methods 2018, 15(11):962-968.

28. Farzaei $\mathrm{MH}$, Bahramsoltani R, Abdollahi M, Rahimi R: The Role of Visceral Hypersensitivity in Irritable Bowel Syndrome: Pharmacological Targets and Novel Treatments. Journal of neurogastroenterology and motility 2016, 22(4):558-574.

29. Smriga M, Torii K: L-Lysine acts like a partial serotonin receptor 4 antagonist and inhibits serotoninmediated intestinal pathologies and anxiety in rats. Proceedings of the National Academy of Sciences of the United States of America 2003, 100(26):15370-15375.

30. Shankar V, Reo NV, Paliy O: Simultaneous fecal microbial and metabolite profiling enables accurate classification of pediatric irritable bowel syndrome. Microbiome 2015, 3:73.

31. Peter J, Fournier C, Durdevic M, Knoblich L, Keip B, Dejaco C, Trauner M, Moser G: A Microbial Signature of Psychological Distress in Irritable Bowel Syndrome. Psychosomatic medicine 2018, 80(8):698-709.

32. Huang F, Zheng X, Ma X, Jiang R, Zhou W, Zhou S, Zhang Y, Lei S, Wang S, Kuang J et al: Theabrownin from Pu-erh tea attenuates hypercholesterolemia via modulation of gut microbiota and bile acid metabolism. Nature communications 2019, 10(1):4971.

33. Clarke G, Fitzgerald P, Hennessy AA, Cassidy EM, Quigley EM, Ross P, Stanton C, Cryan JF, Dinan TG: Marked elevations in pro-inflammatory polyunsaturated fatty acid metabolites in females with irritable bowel syndrome. Journal of lipid research 2010, 51(5):1186-1192. 
34. Berstad A, Raa J, Valeur J: Tryptophan: 'essential' for the pathogenesis of irritable bowel syndrome? Scandinavian journal of gastroenterology 2014, 49(12):1493-1498.

35. Christmas DM, Badawy AA, Hince D, Davies SJ, Probert C, Creed T, Smithson J, Afzal M, Nutt DJ, Potokar JP: Increased serum free tryptophan in patients with diarrhea-predominant irritable bowel syndrome. Nutrition research 2010, 30(10):678-688.

36. Williams BB, Van Benschoten AH, Cimermancic P, Donia MS, Zimmermann M, Taketani M, Ishihara A, Kashyap PC, Fraser JS, Fischbach MA: Discovery and characterization of gut microbiota decarboxylases that can produce the neurotransmitter tryptamine. Cell host \& microbe 2014, 16(4):495-503.

37. Rogers MA, Greene MT, Young VB, Saint S, Langa KM, Kao JY, Aronoff DM: Depression, antidepressant medications, and risk of Clostridium difficile infection. BMC medicine 2013, 11:121.

38. Guo Y, Niu K, Momma H, Kobayashi Y, Chujo M, Otomo A, Fukudo S, Nagatomi R: Irritable bowel syndrome is positively related to metabolic syndrome: a population-based cross-sectional study. PloS one 2014, 9(11):e112289.

39. Lacy BE, Mearin F, Chang L, Chey WD, Lembo AJ, Simren M, Spiller R: Bowel Disorders. Gastroenterology 2016, 150(6):1393-1407.e1395.

40. Lacy BE, Patel NK: Rome Criteria and a Diagnostic Approach to Irritable Bowel Syndrome. Journal of clinical medicine 2017, 6(11).

41. Lin CY, Zhao L, Huang T, Lu L, Khan M, Liu J, Zhong LLD, Cai ZW, Fan BM, Wong AOL et al: Spexin Acts as Novel Regulator for Bile Acid Synthesis. Frontiers in physiology 2018, 9:378.

42. Boughton BA, Callahan DL, Silva C, Bowne J, Nahid A, Rupasinghe T, Tull DL, McConville MJ, Bacic A, Roessner U: Comprehensive profiling and quantitation of amine group containing metabolites. Analytical chemistry 2011, 83(19):7523-7530.

43. Zoetendal EG, Heilig HG, Klaassens ES, Booijink CC, Kleerebezem M, Smidt H, de Vos WM: Isolation of DNA from bacterial samples of the human gastrointestinal tract. Nature protocols 2006, 1(2):870873.

44. Chen Y, Chen Y, Shi C, Huang Z, Zhang Y, Li S, Li Y, Ye J, Yu C, Li Z et al: SOAPnuke: a MapReduce acceleration-supported software for integrated quality control and preprocessing of high-throughput sequencing data. GigaScience 2018, 7(1):1-6.

45. Li R, Yu C, Li Y, Lam TW, Yiu SM, Kristiansen K, Wang J: SOAP2: an improved ultrafast tool for short read alignment. Bioinformatics 2009, 25(15):1966-1967.

46. Truong DT, Franzosa EA, Tickle TL, Scholz M, Weingart G, Pasolli E, Tett A, Huttenhower C, Segata N: MetaPhIAn2 for enhanced metagenomic taxonomic profiling. Nature methods 2015, 12(10):902-903.

47. Segata N, Izard J, Waldron L, Gevers D, Miropolsky L, Garrett WS, Huttenhower C: Metagenomic biomarker discovery and explanation. Genome biology 2011, 12(6):R60.

\section{Figures}


a

W

Discovery cohort

Validation cohort

Fecal Metagenomic

shotgun sequencing

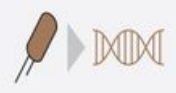

Fecal MicroBiome

b

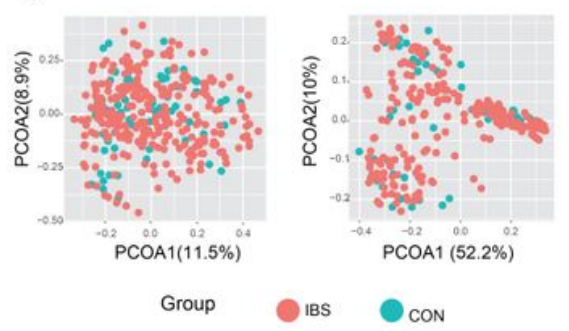

IBS

264

86

Fecal Metabolome LC-MS

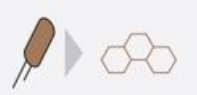

Fecal MetaBolome

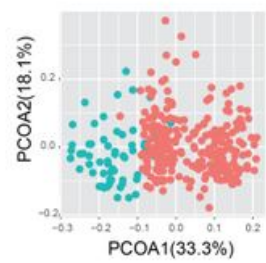

c

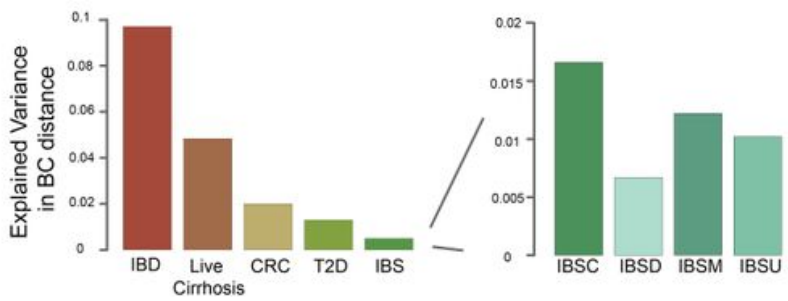

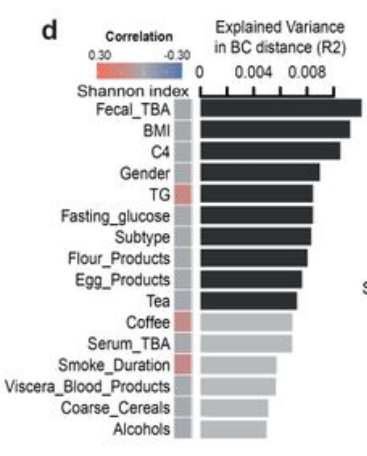

Fecal microbiome

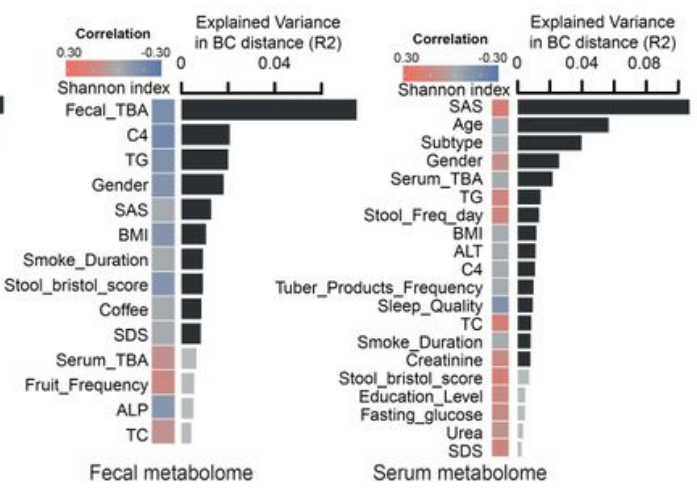

\section{Figure 1}

Multi-omics profiles of individuals with IBS (a) Metagenomic and metabolic sequencing of two IBS cohorts: a 330 members discovery cohort and a 101 members validation cohort. (b). Principal coordinates analysis (PCOA) of discovery cohort individuals based on gut microbiota, fecal metabolome and serum metabolome profiles, respectively (Bray-Curtis distance). (c). Comparisons of gut microbiota dysbiosis in different diseases and different subtypes of IBS. (d). Shown are associated phenotypes for gut microbiota composition, fecal metabolome and serum metabolome data. Black bars indicate statistical significance $(\mathrm{FDR}<0.1)$. The heatmap indicates significant positive correlations (red) or negative correlations (blue). 


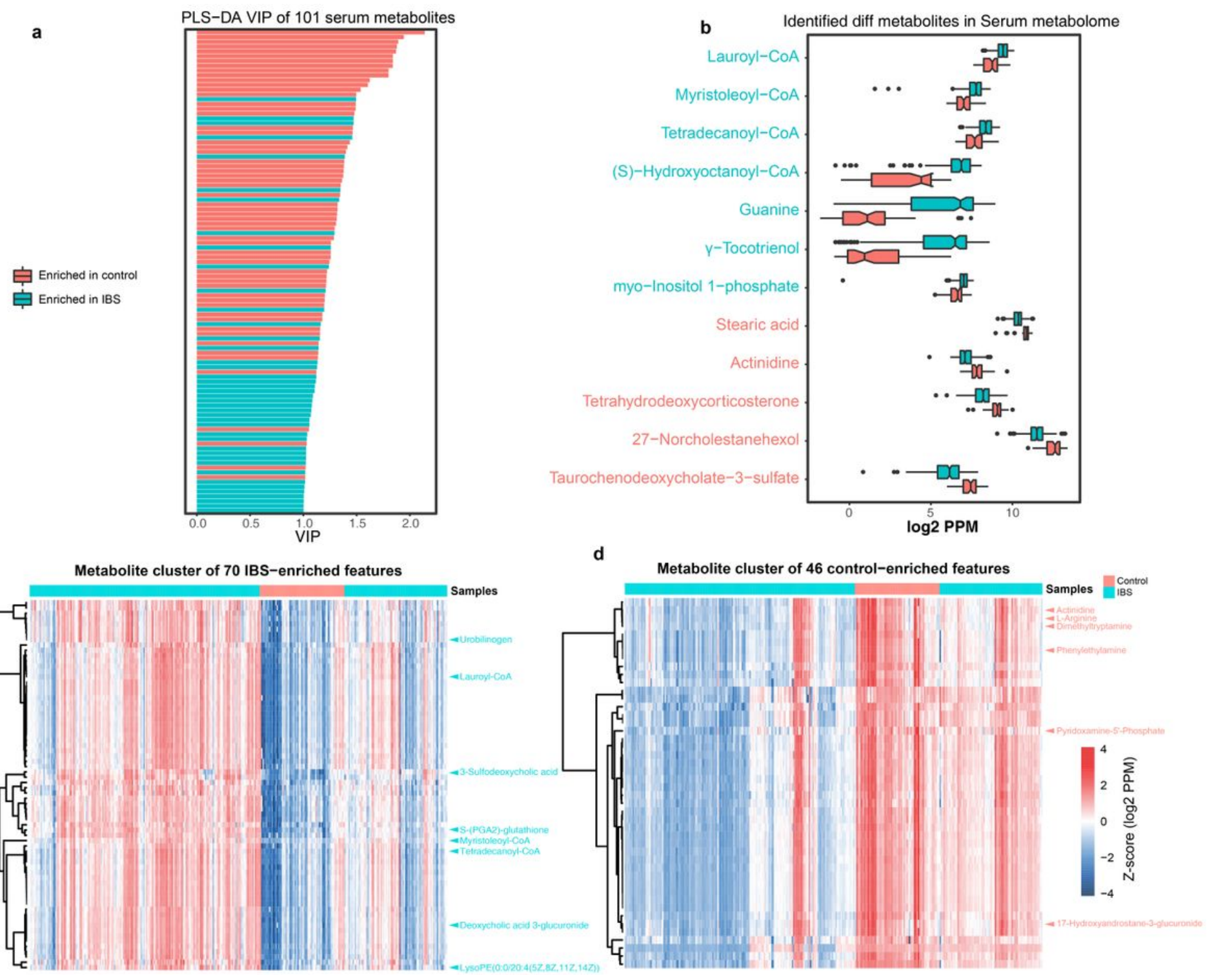

Figure 2

Differentially abundant metabolites and clusters. (a) Barplot shows the PLS-DA variable importance (VIP) results in projection of 101 structurally identified differentially abundant serum metabolites. (b) Comparison of the abundance of identified metabolites significantly differed between controls and IBS. Boxplot 'boxes' indicate the first, second and third quartiles of the data, with points outside the inner fences plotted as outliers. (c) The largest cluster contains 70 metabolites, and all of them significantly elevated among IBS patients. (d) The cluster contain 46 metabolite features, and all of them significantly elevated in controls. This cluster was enriched for a variety of amines. Abundances are in logtransformed PPM units. 
a
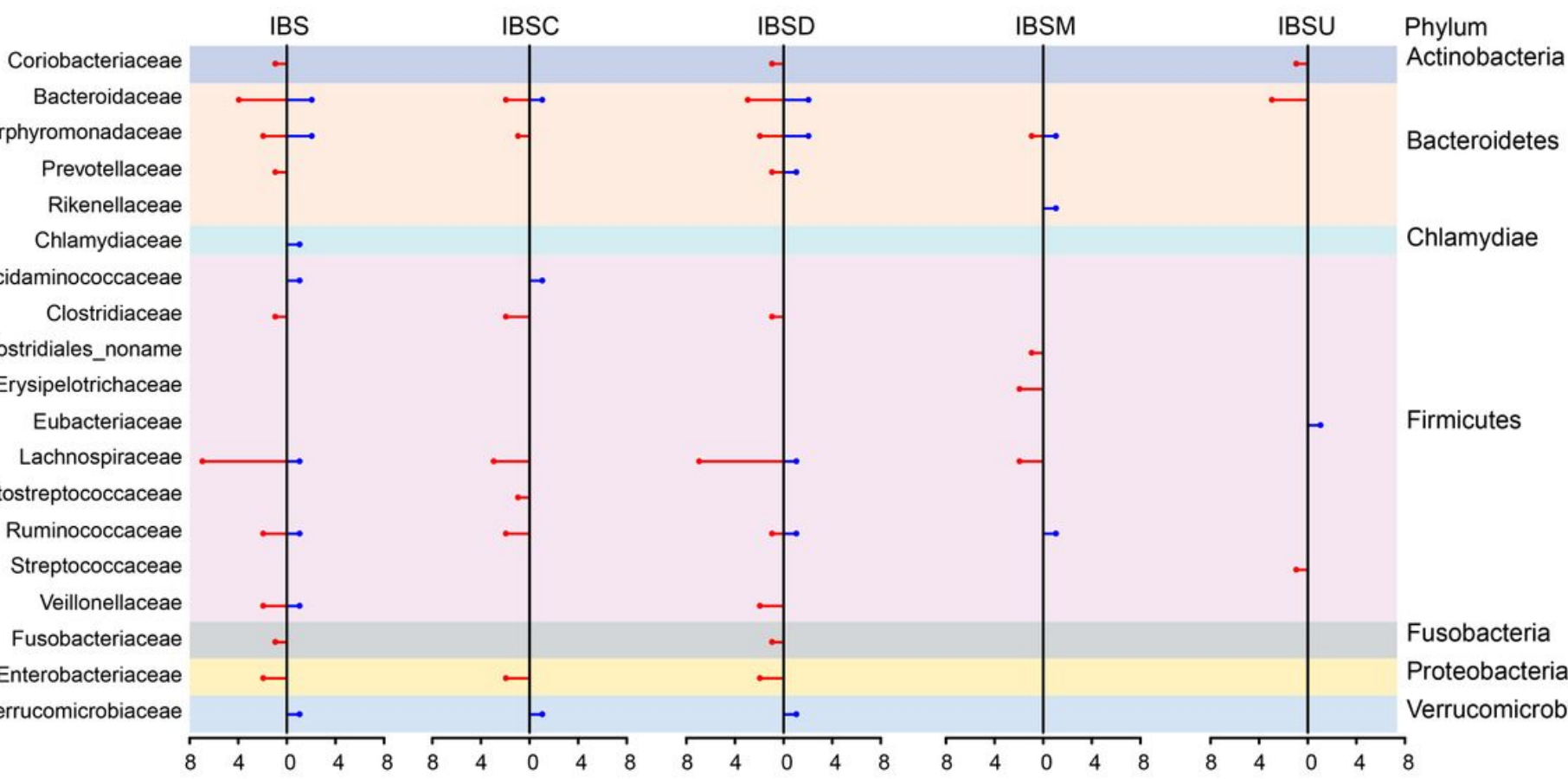

b

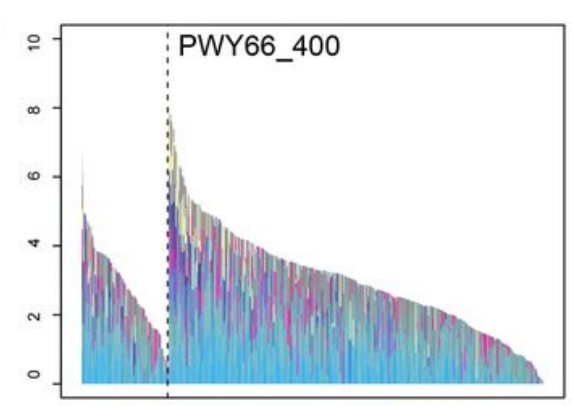

e

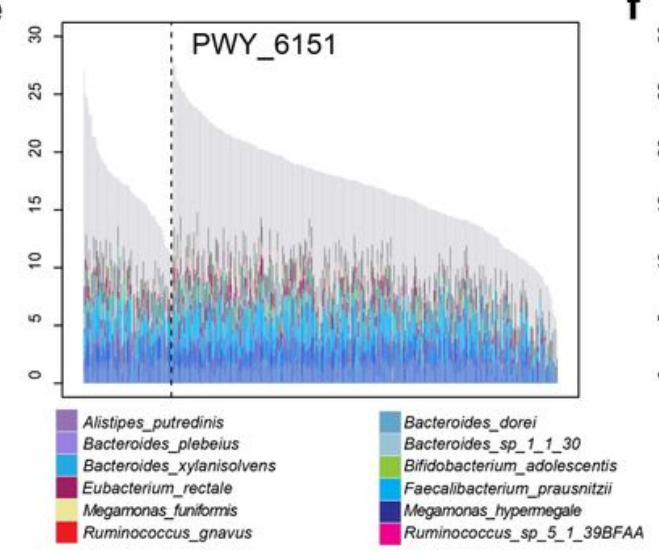

C

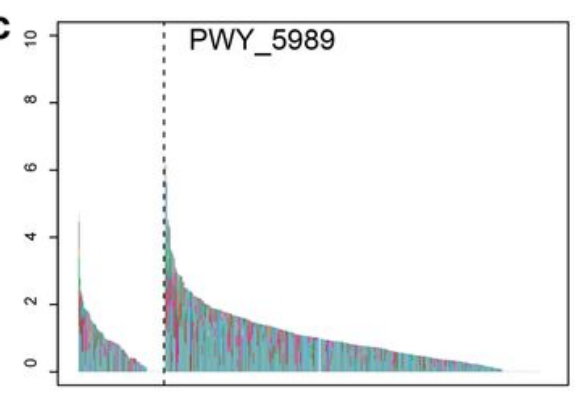

f

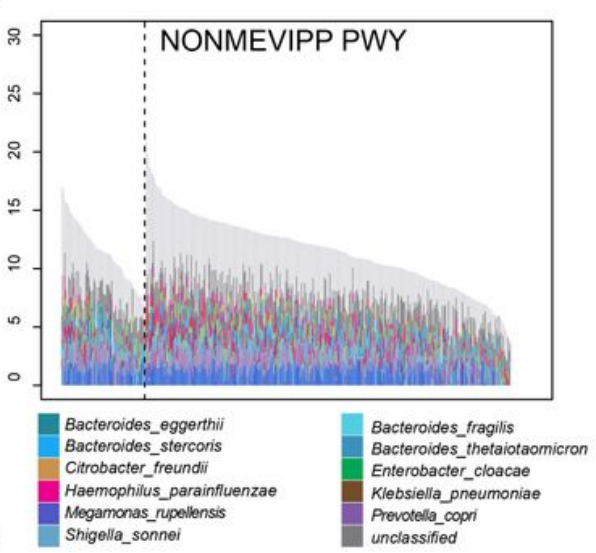

d

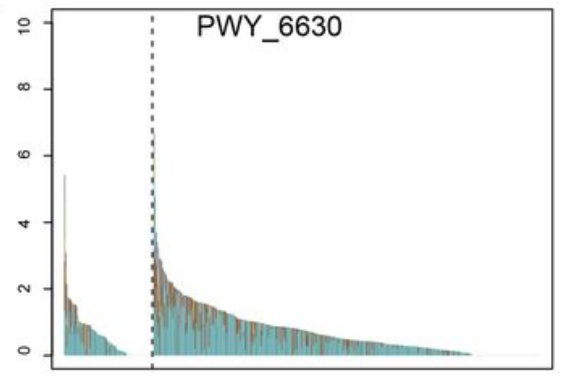

$\mathbf{g}_{8}$

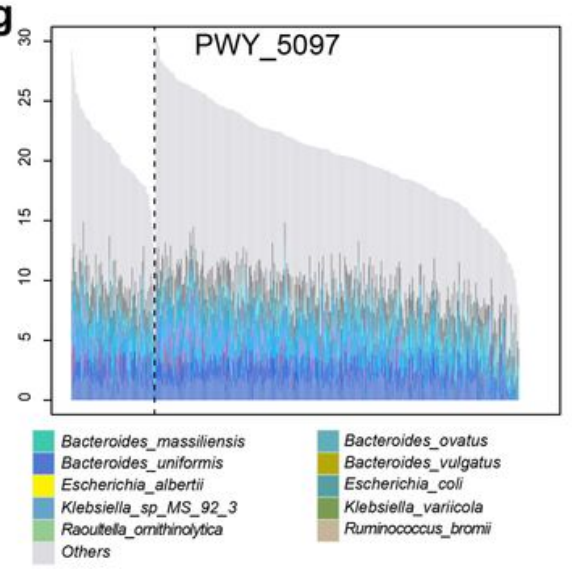

Figure 3

Gut microbiota divergency between IBS and healthy controls (a) Statistically significant species were shown at family level. Orange color represents species that were higher in IBS whereas blue color represents species that were lower in IBS. (b-g) Examples of MetaCyc pathways that were differentially abundant in IBS, annotated by their taxonomic contributors (b,c,d are enriched in IBS; e,f,g are depleted). 


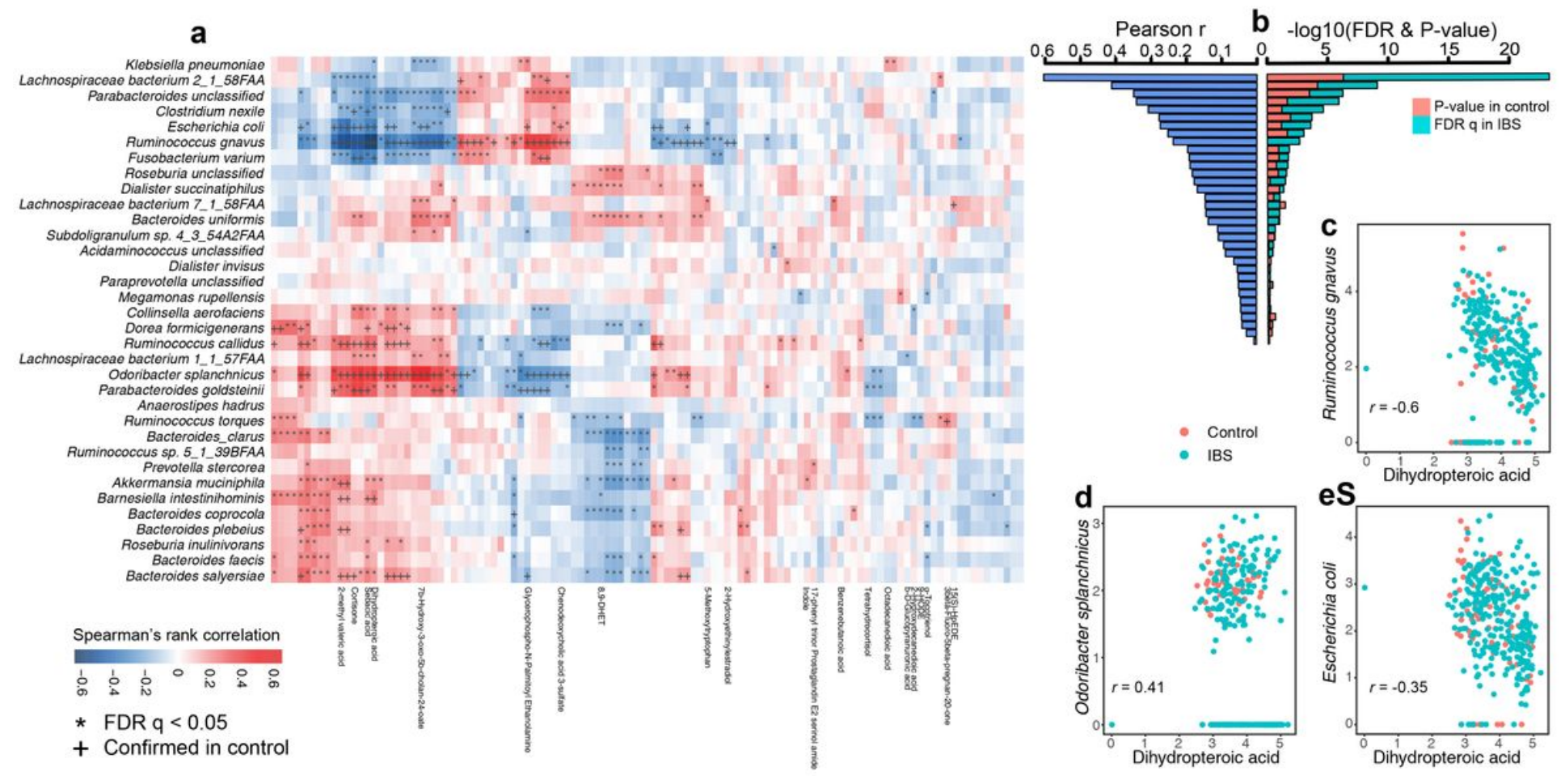

Figure 4

Association between Fecal metabolites and gut microbiota. (a) Association between 34 differentially abundant species and 21 known fecal metabolites. (b) Pearson correlation, q-value in IBS and p-value in controls between dihydropteroic acid and 33 species. (c-e) The top 3 species associated with dihydropteroic acid. Values plotted are log10-transformed. 
a

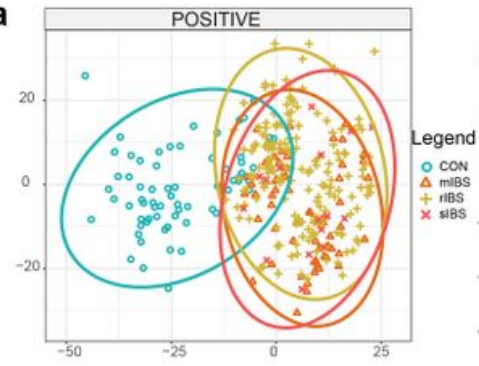

b
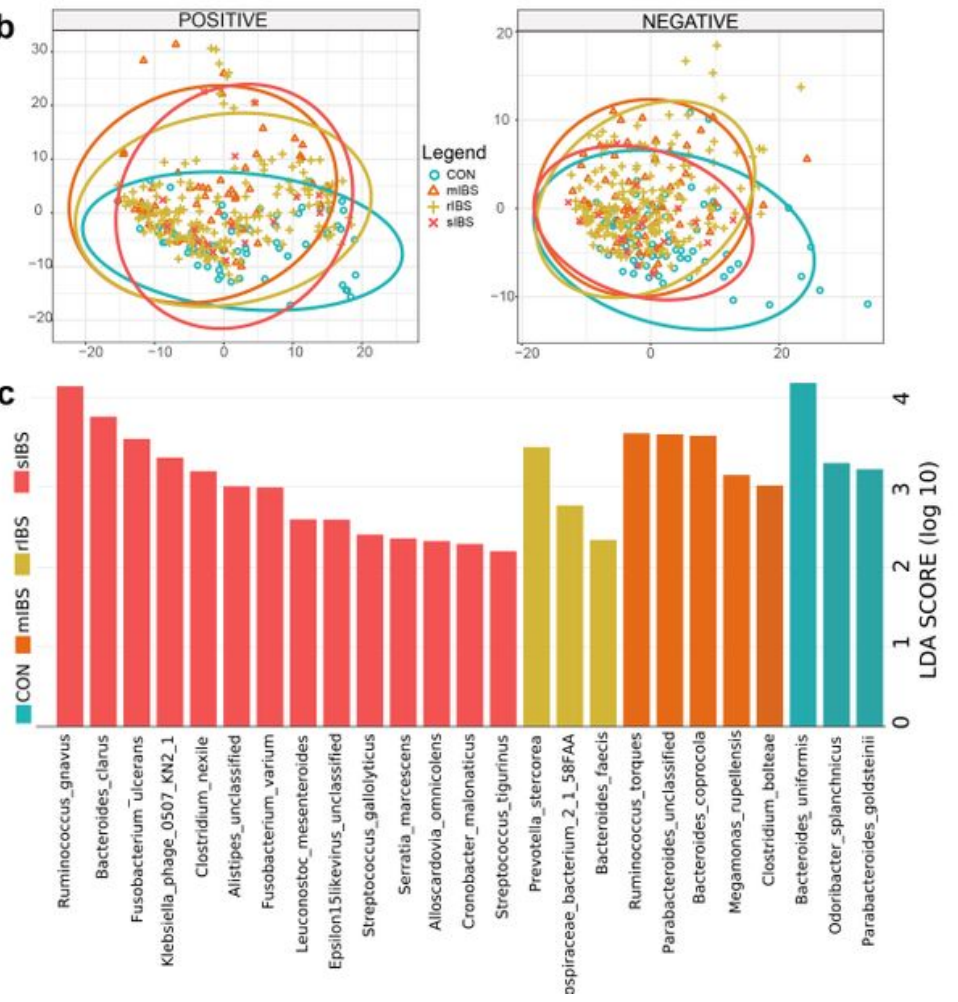

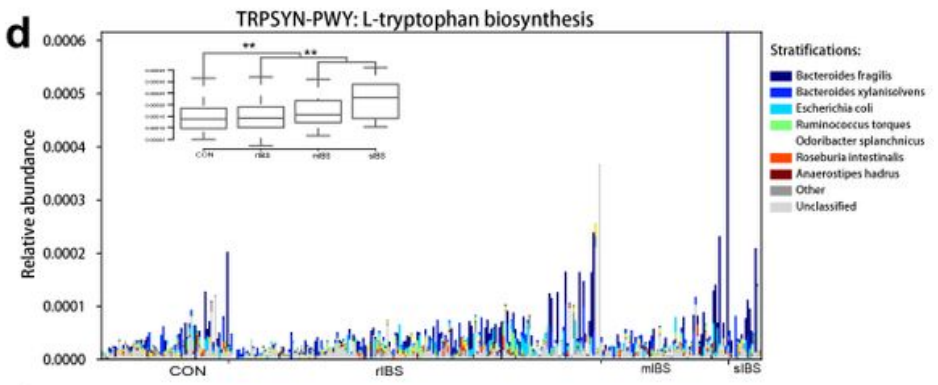

e

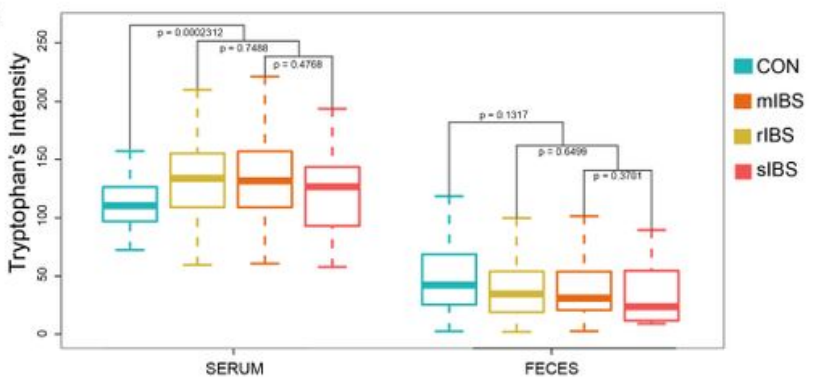

Figure 5

Aberrant metabolic and microbiome patterns in IBS patients with depression. (a) PLS-DA score plots based on the metabolic profiles in serum metabolome data from control, rIBS, mIBS, and sIBS group in positive and negative ion mode. (b) PLS-DA score plots based on the metabolic profiles in fecal metabolome data from control, rIBS, mIBS, and sIBS group in positive and negative ion mode. (c) The significantly altered species across healthy controls and patients. (d) The elevated L-tryptophan biosynthesis pathway in healthy controls and patients with mIBS/sIBS. (e) The signal intensity of tryptophan in serum and feces using targeted metabolome technology. 
a

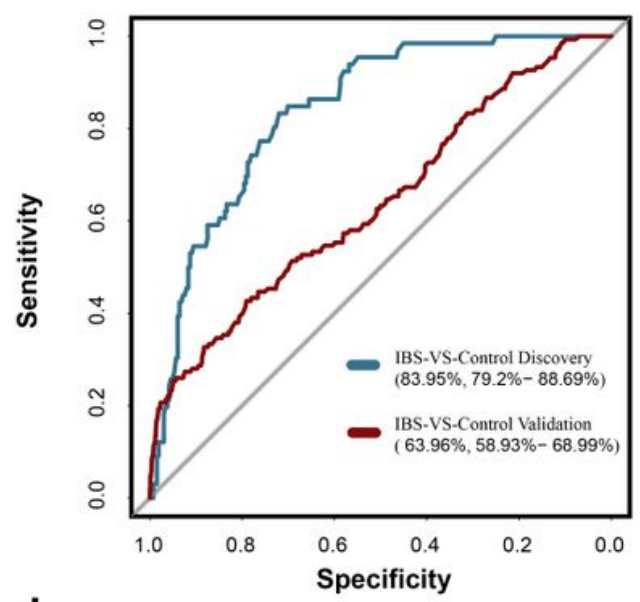

d

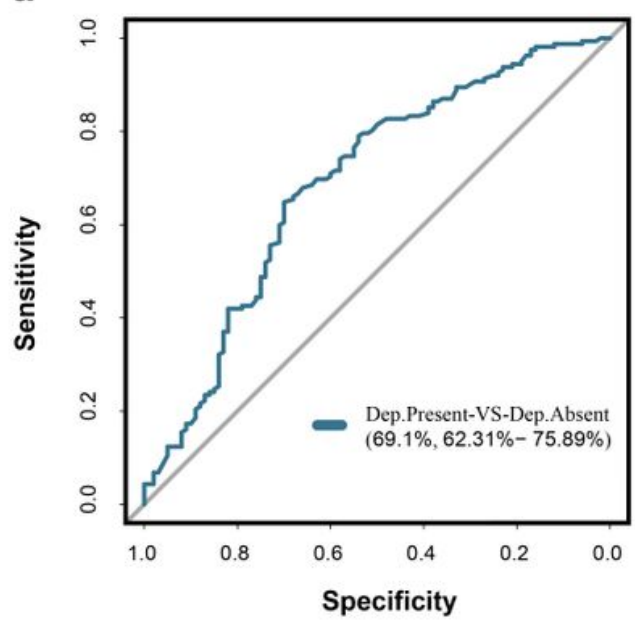

b

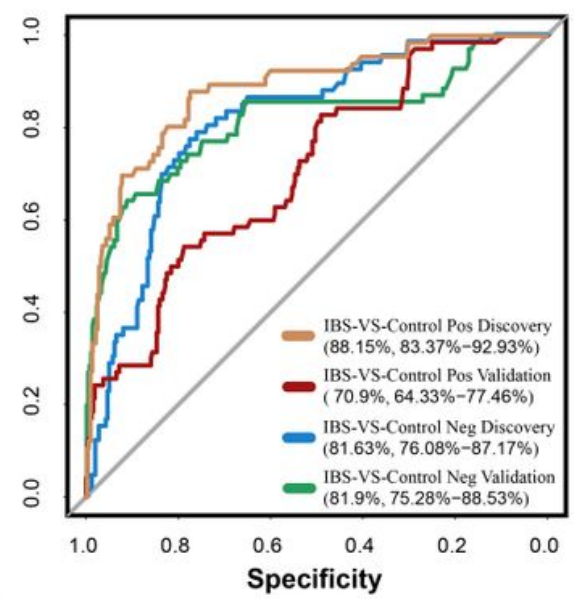

e

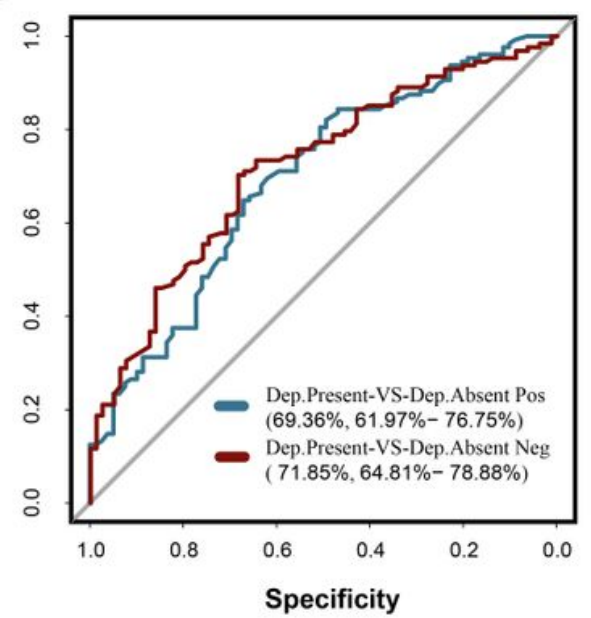

C

Serum Metabolome Data

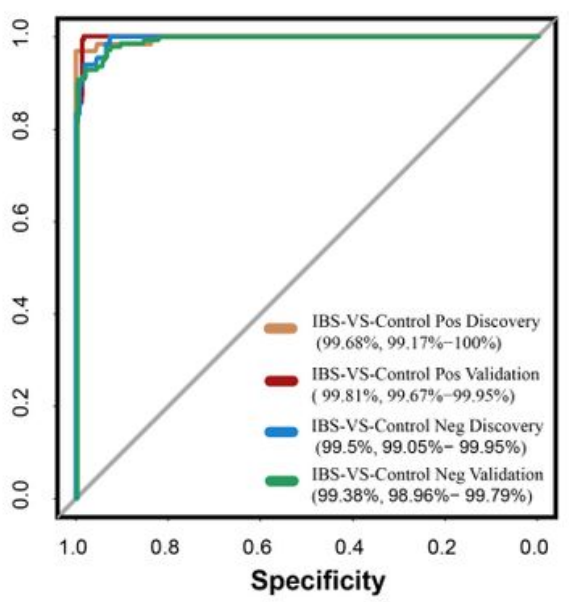

f

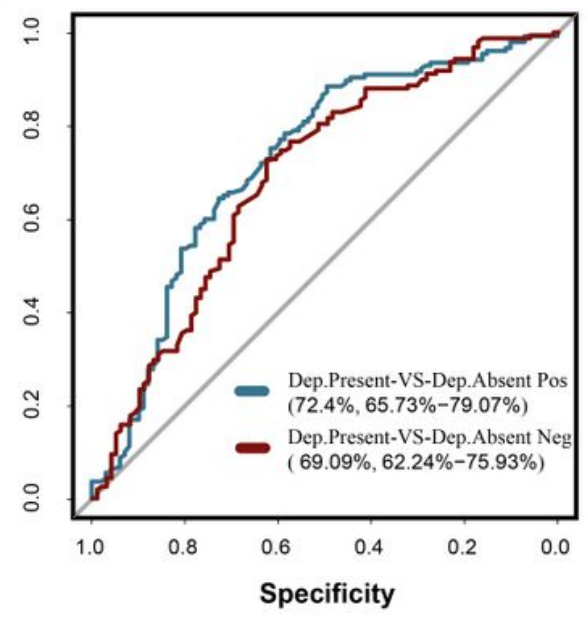

Figure 6

Using multi-omics data to predict different IBS symptoms. (a) Receiver operating characteristic curve (ROC) of using microbiota to classify IBS from control. For serum metabolome data and fecal metabolome, only the possibility distribution derived from untargeted positive ion mode is presented. (b) ROC of using fecal metabolome to classify IBS from control in discovery and validation cohorts. (c) ROC of random forest model using serum metabolome data to classified IBS from control in discovery and validation cohorts. AUC value is presented in the format of $95 \%$ confident interval. (d-f) Receiver operating characteristic curve (ROC) of training and predicting depression status of IBS patients using microbiota, fecal and serum metabolome data, respectively.

\section{Supplementary Files}

This is a list of supplementary files associated with this preprint. Click to download.

- SupplementaryTable15.xlsx

- SupplementaryTable14.xlsx

- SupplementaryTable13.xlsx 
- SupplementaryTable12.xlsx

- SupplementaryTable11.xlsx

- SupplementaryTable10.xlsx

- SupplementaryTable9.xIsx

- SupplementaryTable8.xIsx

- SupplementaryTable7.xIsx

- SupplementaryTable6.xlsx

- SupplementaryTable5.xlsx

- SupplementaryTable4.xlsx

- SupplementaryTable3.xlsx

- SupplementaryTable2.xlsx

- SupplementaryTable1.xlsx

- SupplementaryFigure13.pdf

- SupplementaryFigure12.pdf

- SupplementaryFigure11.pdf

- SupplementaryFigure10.pdf

- SupplementaryFigure9.pdf

- Supplementaryinformation.docx

- SupplementaryFigure8.pdf

- SupplementaryFigure7.pdf

- SupplementaryFigure6.pdf

- SupplementaryFigure5.pdf

- SupplementaryFigure4.pdf

- SupplementaryFigure3.pdf

- SupplementaryFigure2.pdf

- SupplementaryFigure1.pdf 